\title{
Mapping Dielectric Properties with Torsionally Stabilized Nano Impedance Microscopy: Hard Materials to Biomolecules
}

\author{
Kendra Kathan-Galipeau, Xi Chen, Bohdana Discher, and Dawn A. Bonnell* \\ Nano/Bio Interface Center, The University of Pennsylvania, Philadelphia, PA 19104 \\ *bonnell@lrsm.upenn.edu
}

\section{Introduction}

During the last twenty-five years of scanning tunneling microscopy (STM), atomic resolution has become routine in studies of a wide range of materials. While less routine, atomic force microscopy (AFM) also can achieve atomic resolution, even on insulating surfaces. These two microscopy approaches have significantly advanced our understanding of the surface physics and chemistry of many important phenomena. A significant contribution of scanning probe microscopy is that it can characterize local properties as well as structure. Surprising levels of spatial resolution have been achieved in scanning probes that can access continuum properties such as resistance, capacitance, etc. $[1,2]$.

Recently there has been a focus on extending AFM to examine more complex properties, such as dielectric function and electromechanical coupling, while pushing the limits of spatial resolution of properties ever further. In this paper we describe a new scanning probe microscopy method that accesses local impedance, leading to information about dielectric function and polarization that can be applied to both hard and soft materials. Combining this with local laser excitation yields quantitative information about excited states and photo conduction.

\section{Scanning Probes and Impedance}

Most force-based scanning microscopes that probe electronic properties employ a conducting tip and the application of an electrical signal to the tip-surface junction. In some cases this is done with the probe tip in contact with the surface, yielding, for example, resistance, capacitance, and piezo-force microscopies. In other cases, the tip is above the surface leading to potentiometry and Kelvin probe microscopy. Introducing frequency variation into these local electrical measurements allows the access of increased information about the sample, in the present case dielectric function. Scanning Impedance Microscopy (SIM) can be done in non-contact mode, where the voltage modulation is across the sample of interest [3]. Alternatively, it can be done in contact or near contact mode, which is analogous to macroscopic impedance spectroscopy and is referred to as nano impedance microscopy/ spectroscopy (NIM/NIS) [4, 5].

Impedance spectroscopy has long been recognized as one of the major techniques for the characterization of $\mathrm{AC}$ transport in materials. Impedance is defined as the ratio of the AC voltage excitation to a current response: $Z(\omega)=$ $V(\omega) / I(\omega)$. Impedance, $Z$, is usually expressed in complex form in terms of its modulus, $|Z|$, and phase, $\theta$, as $Z=|Z| e^{i \theta}$. Capacitance, resistance, and associated frequency dependence of the impedance depend on the material properties. Typical applications of impedance spectroscopy are to differentiate grain boundary, grain interior, and electrode impedances by fitting the data to corresponding equivalent circuit models [6]. However, the general limitation of all macroscopic techniques is that only average transport properties are determined. The first SPM techniques for accessing AC transport behavior were SIM [3, 4, 6-11] and NIM [4, 7]. Strategies to enhance the sensitivity of these measurements have been suggested [4, 12-19].

These approaches are facilitating examinations of a range of material systems and devices. A limitation, however, is that they cannot be applied to soft surfaces, such as organic or biomolecular monolayers. The challenges are to control the tip-surface force so as not to damage the surface, to detect very small signals, and to eliminate the contributions from stray capacitances. This is accomplished in torsionally stabilized nano impedance microscopy (TR-NIM), where we introduce sample-tip laser excitation to increase the property information content.

\section{Torsionally Stabilized Nano Impedance Microscopy}

Torsional resonance is employed to control the sample-tip force interaction and topographic imaging, enabling near-field contact with the lowest possible force $(10 \mathrm{nN})$. In torsional mode, the probe is oscillated along the cantilever's long axis, creating a rotational oscillation. An impedance measurement system is designed to: operate at very low currents typical for SAMs $(<1 \mathrm{pA})$, operate over a significant frequency range $(\mathrm{kHz}$ to $100 \mathrm{kHz}$ ) to increase signal-to-noise ratios, and compensate for system stray capacitances.

Figure 1 illustrates a schematic diagram of the TR-NIM configuration. The tip is maintained in proximity of the sample surface by the microscope controller. The electrical impedance of tip-surface junction is measured using external circuitry consisting of a current amplifier, signal generator, and lock-in amplifier. Tunneling currents across biological molecules are quite small; to measure this current, a high-speed, high-gain current amplifier is required. The signal generator and current amplifier have a common ground. A reference signal is fed into the lock-in amplifier for synchronization of signal generator and lock-in amplifier. The signal from the amplifier is summed with the compensating signal to account for the capacitance of the entire system. This then enters the lock-in amplifier. The output of the lock-in amplifier is fed into the microscope controller for analysis. The spatial resolution can be estimated to be about $20 \mathrm{~nm}$. The resolution of capacitance is $2 \mathrm{aF}$.

The total impedance can be modeled as the impedance due to capacitive coupling, which is the impedance due to 


\section{A perfect 10-9}

\section{$\ldots$ is only possible with perfect EM sample preparation.}

No matter what type of imaging instrument you use; TEM, SEM, LM, Confocal or AFM, the ultimate quality of the image comes from high-quality sample preparation. Leica Microsystems has a full range of innovative instrumentation to deliver perfect preparation for all sample materials.

- Sectioning

- Planing

- Contrasting

- Coating and Drying
-Processing

- Target Polishing

- High Pressure Freezing
-Staining

- Ion Milling

- Cryoprocessing and Transfer

A perfect $10^{\circ}$ ! See the difference Leica Microsystems quality can make for you.

Visit www.leica-microsystems.com/yourimage

\section{Living up to Life}

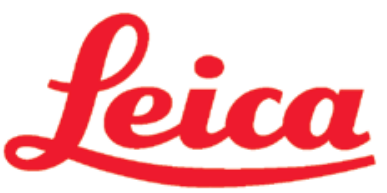

MICROSYSTEMS 
a)

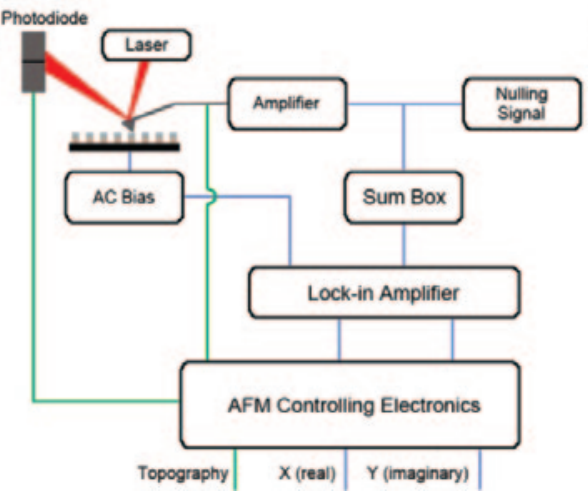

b)

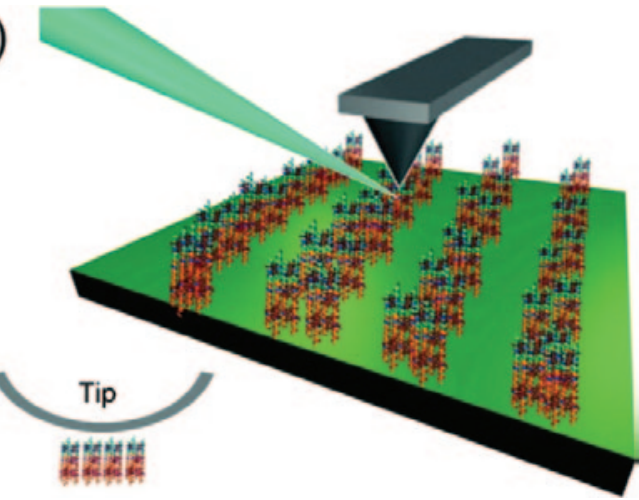

Figure 1: Torsional resonance nanoimpedance microscopy (TR-NIM) schematic diagram (a). An AC bias is applied to the tip/sample, and the alteration in this signal due to the impedance of the sample is output to the amplifier. The signal from the amplifier is summed with the compensating signal to account for the capacitance of the entire system. This combined signal then enters the lock-in amplifier. The output of the lock-in is fed into the microscope controller for analysis. (b) Schematic illustration of the tip-surface junction allowing photo excitation of patterned protein layers. (b) is from Kathan-Galipeau et al., ACS Nano 4835, copyright 2011, with permission of ACS.

the system and the impedance of the sample. Given the film size and properties, a significant contribution to the signal is noise from the system's capacitive coupling. There is capacitive coupling related to the cantilever, cantilever holder, and other structural components of the AFM. Therefore, the nulling signal is used to account for most of the effect of these other signals. It is assumed that the impedances of the graphite and sample are in parallel.

Assuming the molecule can be represented as an RC circuit [20],

$$
R=\frac{10 \sqrt{2} V G}{s X} \text { and } C=\frac{s Y}{10 \sqrt{2} V G \omega}
$$

where $R=$ resistance, $C=$ capacitance, $V=$ applied voltage, $\omega=$ frequency, $s=$ sensitivity of lock-in amplifier, $G=$ gain of current amplifier, and $X$ and $Y=$ output of lock-in amplifier. ( $\sqrt{2}$ is required because $V_{\mathrm{o}}$ is an RMS voltage; 10 is a conversion factor within the lock-in amplifier.) The values for $X$ and $Y$ are relative to that of the graphite. If the compensating signal is perfect, we measure zero impedance over graphite. The change in impedance is then entirely attributed to the molecule.

\section{Materials and Methods}

For TR-NIM experiments, a platinum-iridium coated tip was used; $f_{o}=146-236 \mathrm{kHz}$ and $k=21-98 \mathrm{~N} / \mathrm{m}$ (Nanosensors). The AFM instrumentation was performed on a Veeco Dimension 3100 with a IVa controller. Two current amplifiers were used, which together provide a gain range of $1 \times 10^{2}-1 \times 10^{11}$ V/A (Femto DLPCA-200, DHPCA-100) Two lock-in amplifiers provided a frequency range from $1 \mathrm{mHz}-200 \mathrm{MHz}$ (SRS830, SRS844). The AC signal can be applied in a range from $1 \mu \mathrm{Hz}$ to $30 \mathrm{MHz}$ (SRS DS345). The sum box has a range from $0.002-60 \mathrm{MHz}$ (Mini-Circuits ZSC-2-2).

\section{TR-NIM on Hard Materials}

To demonstrate the effectiveness of TR-NIM for probing dielectric behavior, Figure 2 shows a typical TR-NIM image of CdTe. Figure 2 shows the topography, amplitude, and phase of impedance (left to right). At $70 \mathrm{kHz}$, the resistance is $8035 \mathrm{k} \Omega$, and the capacitance is $21.3 \mathrm{fF}$. A dielectric constant of 10.1 was determined from these images, assuming $1 \mathrm{~mm}$ thick CdTe and a $8.7 \mathrm{~nm}$ tip size. In comparison, the literature value of the dielectric constant of CdTe is 10.9 [21]. This agreement provides confirmation of the effectiveness of TR-NIM for determining the dielectric constant of materials.

\section{TR-NIM on Soft Materials: Biomolecules Toward Protein-Based Circuits}

Recently, the appreciation of the high energy yield and quantum efficiencies of a number of natural photo-activated proteins, in the context of a decade of research on molecular electronics, has raised the possibility of new nanoelectronic
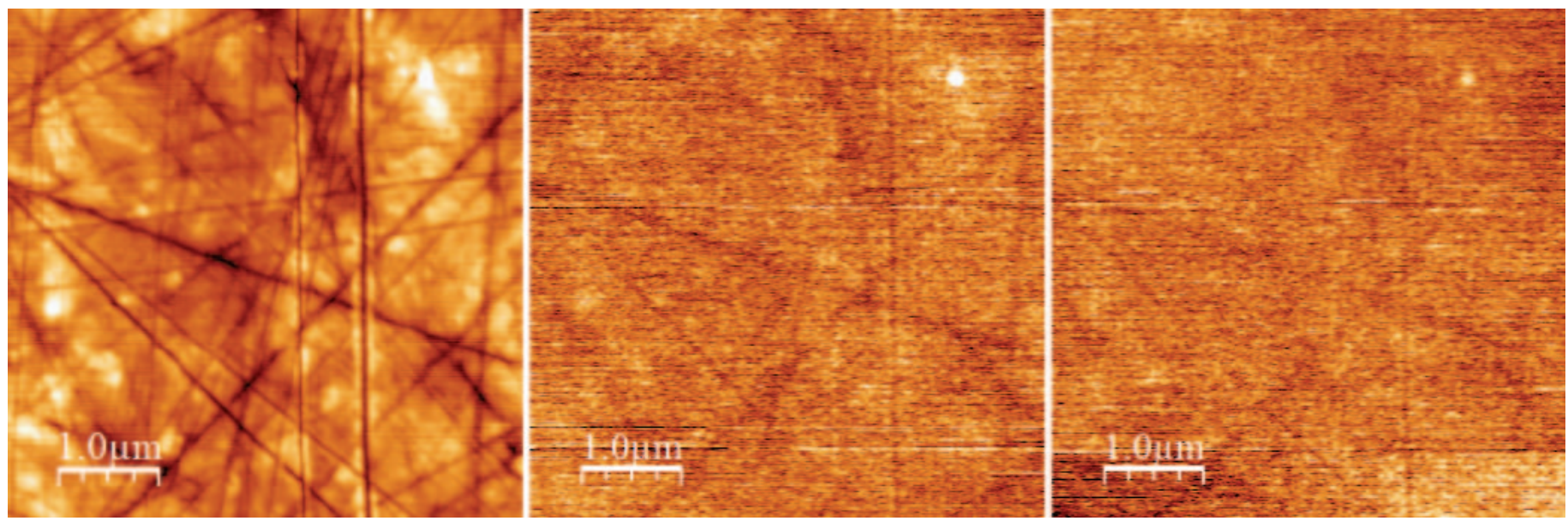

Figure 2: TR-NIM image of CdTe $\langle 0001\rangle$ topography, amplitude, and phase of impedance (left to right). Height $z$-range $=15 \mathrm{~nm}$, amplitude range $=80 \mathrm{mV}$, phase range $=120 \mathrm{mV}$. Parameters: $70 \mathrm{kHz}, 0.5 \mathrm{~V}$ applied signal, gain $=10^{9}$, sensitivity $=5 \mathrm{mV}$. Platinum-iridium coated cantilevers were used with the following properties: resonant frequency $=146-236 \mathrm{kHz}$, spring constant $=21-98 \mathrm{~N} / \mathrm{m}$, and tip radius $\sim 40 \mathrm{~nm}$. 
device applications. Bio-based strategies for light-activated electron pumps, solar cells, chemical sensors, and optoelectronics have been suggested. An obvious challenge is to understand and control the properties of the proteins when attached to electrodes and configured in the ambient environments relevant to potential device architectures. Despite the challenges of characterizing biomolecule interfaces that are a consequence of the delicate nature of the structure, probing a complex property such as impedance would provide valuable insight.

The application of TR-NIM is illustrated here to determine the resistance, capacitance, and dielectric properties of novel optically active biomolecules [19]. These molecules consist of tetramers of alpha helix polypeptides and provide a convenient functional alternative to natural proteins. We recently studied the behavior of zinc protoporphyrin IX (ZnPP) [19]. To determine the behavior of these functional biomolecules on electrodes, microcontact printing was used to create stripes that alternate between $\mathrm{ZnPP}$ maquettes and bare graphite. Understanding the dielectric constant is of particular interest because it gives information about the polarizability of these molecules. With a view toward understanding the behavior of future devices, optoelectronic properties of the biomolecules should be probed. This can be accomplished by combining TR-NIM with in-situ laser excitation.
Idealized protein-electrode junction devices can be fabricated by microcontact printing self-assembled layers of proteins in patterns on atomically smooth highly oriented pyrolytic graphite (HOPG) [22]. The first layer of these engineered amphiphilic proteins will orient on the hydrophobic graphite with the hydrophobic domain nearest the graphite due to hydrophobic interactions. Figure 3 presents a typical spatially resolved impedance measurement [19]. The topographic structure shown in Figures $3 \mathrm{a}$ and $3 \mathrm{~b}$ are typical of a protein-patterned electrode, in this case with a pattern pitch of $1 \mu \mathrm{m}$ and protein lines varying in height from 6-20 nm. Many regions of the patterns exhibit heights very close to $6.6 \mathrm{~nm}$, the length of a single 4-helix bundle. These regions, in which the orientation of the molecule with respect to the substrate can be assigned, are identified by height profiles in the range of $6.6 \pm 0.5 \mathrm{~nm}$, and the impedance is analyzed. Figure $3 \mathrm{c}$ identifies several such regions. The component of the impedance related to resistance is mapped in $3 \mathrm{~d}$, and the component related to capacitance is mapped in 3e. Measurements made during laser excitation with $425 \mathrm{~nm}$ light were similar to Figures $3 \mathrm{~d}$ and $3 \mathrm{e}$ but differed in magnitude.

Figure 4 compares several hundred values of the resistance (a) and capacitance (b) in a region of the $\mathrm{ZnPP}$ monolayer with and without laser excitation. This type of analysis enables conclusions to be drawn from small signals with statistical
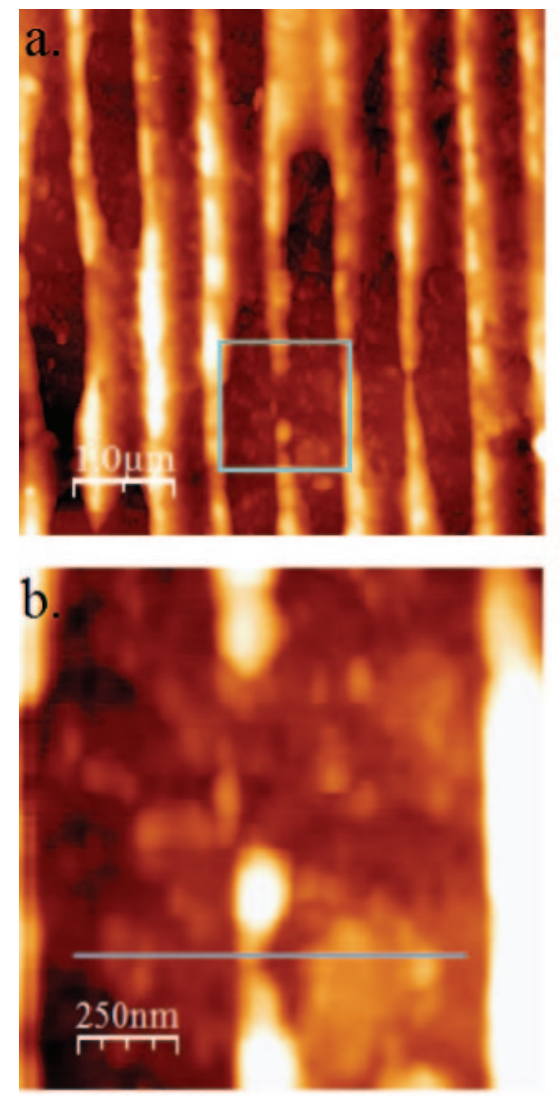
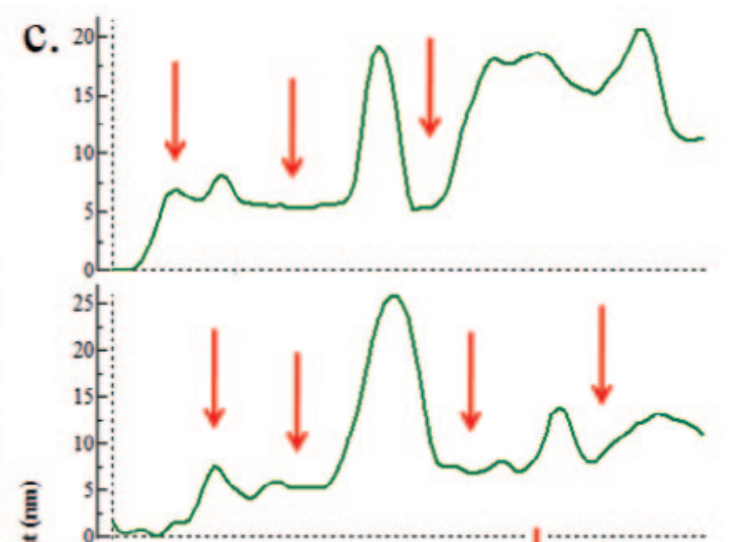

农

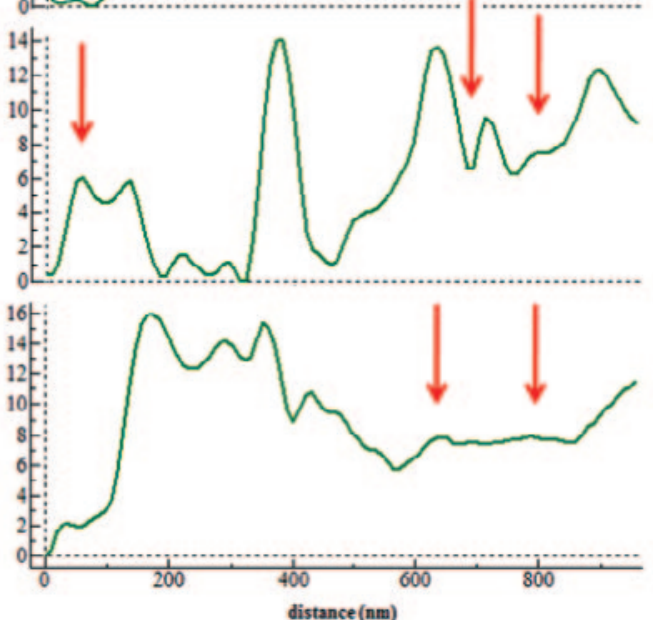

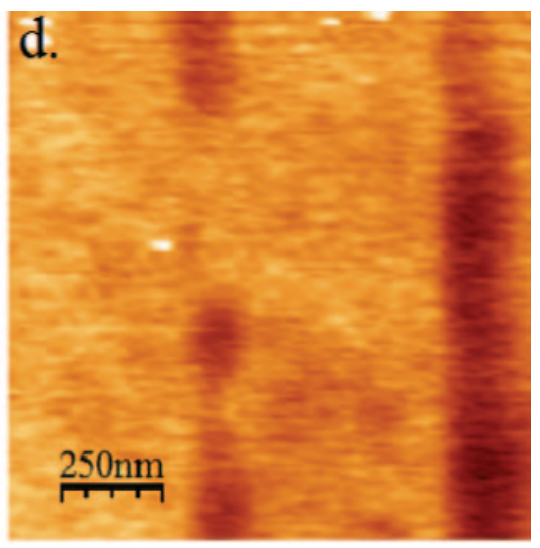

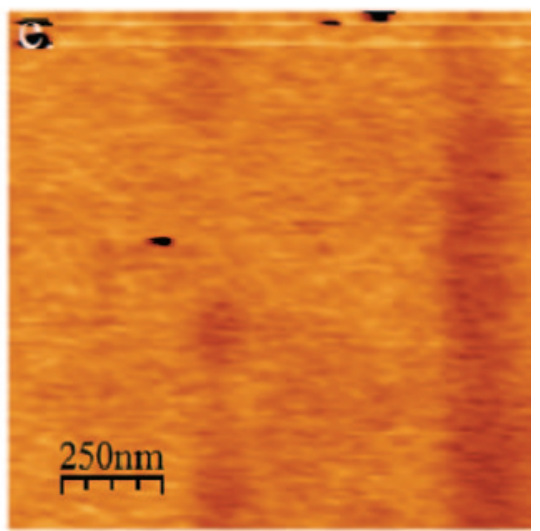

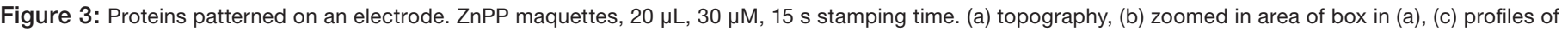

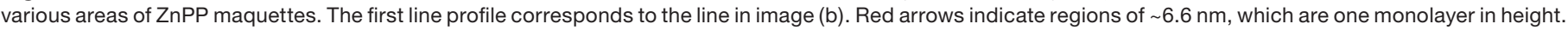

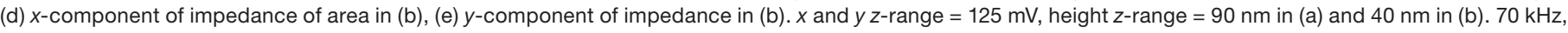
$0.5 \mathrm{~V}$ applied signal. Kathan-Galipeau et al., ACS Nano 4835, copyright 2011, with permission of ACS. 

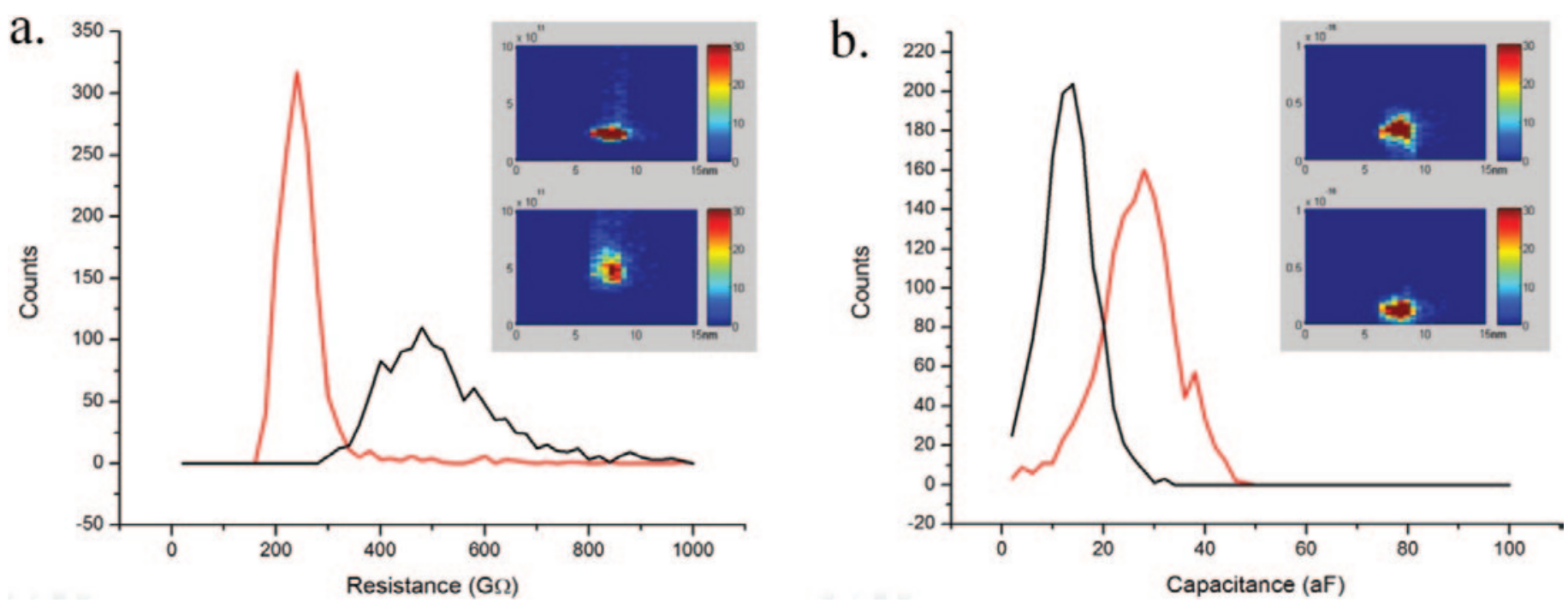

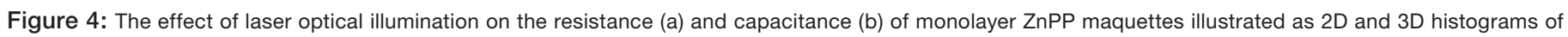

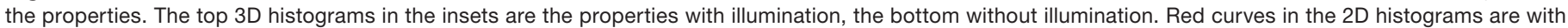
illumination; black curves are without exposure. I = $425 \mathrm{~nm}$. Kathan-Galipeau et al., ACS Nano 4835, copyright 2011, with permission of ACS.

significance. In this case the reduction of resistance and increase in capacitance in the presence of the laser illumination is clear. For these proteins the electronic transport mechanisms are complex, but the capacitance differences can be understood in terms of the polarization in the porphyrin complexes within the protein. The dielectric constant determined from capacitance can be related to the polarization volume in the molecule. When the light is absorbed, the excited electron resides in a more delocalized orbital. The difference in the delocalization and the degree of coupling between the porphyrins can be compared from these measurements.

\section{Summary}

The examples presented illustrate how TR-NIM can be used to determine properties of hard and soft materials. It is an advance over earlier probe-based impedance imaging in that it can characterize soft materials such as organic monolayers and engineered proteins. The approach opens a pathway to bringing frequency and time-dependent probes to bear on questions of the behavior of complex molecules, such as proteins. Accessing the real and imaginary components of properties adds a new dimension of information at the local scale that will be valuable in the development of hybrid devices.

\section{Acknowledgments}

This research was supported by the Nano/Bio Interface Center through the National Science Foundation NSEC DMR08-32802

\section{References}

[1] D Bonnell, ACS Nano 2 (2008) 1753-59.

[2] M Brukman and D Bonnell, Phys Today 61 (2008) 36-42.

[3] SV Kalinin and DA Bonnell, J Appl Phys 91 (2002) 832-39.

[4] R Shao, SV Kalinin, and DA Bonnell, Appl Phys Lett 82 (2003) 1869-71.

[5] R O'Hayre, G Feng, WD Nix, and FB Prinz, J Appl Phys 96 (2004) 3540-49.

[6] SV Kalinin and DA Bonnell, Materials Research Society Symposium Proceedings (2002) 699.
[7] D Bonnell, Scanning Probe Microscopy and Spectroscopy: Theory, Techniques, and Applications, Wiley-VCH, New York, 2001.

[8] BG Buss, DN Evans, H Liu, T Shang, TE Oliphant, SM Schultz, and AR Hawkin, Sens Actuators, A 137 (2007) 338-44.

[9] J Shin, V Meunier, AP Baddorf, and SV Kalinin, Appl Phys Lett 85 (2004) 4240-42.

[10] SV Kalinin, R Shao, and DA Bonnell, J Am Ceram Soc 88 (2005) 1077-98.

[11] SV Kalinin, DA Bonnell, M Freitag, and AT Johnson, Appl Phys Lett 81 (2002) 5219-21.

[12] LSC Pingree, EF Martin, KR Schull, and MC Hersam, IEEE Transactions on Nanotechnology 4 (2005) 255-59.

[13] LSC Pingree and MC Hersam, Appl Phys Lett 87 (2005) 233117.

[14] LSC Pingree, MT Russell, BJ Scott, TJ Marks, and MC Hersam, Org Electron 7 (2007) 465-79.

[15] BJ Rodriguez, S Jesse, K Seal, AP Baddorf, and SV Kalinin, J Appl Phys 103 (2008) 014306.

[16] L Fumagalli, G Ferrari, M Sampietro, I Casuso, E Martinez, J Samitier, and G Gomila, Nanotechnology 16 (2006) 4581-87.

[17] L Fumagalli, G Ferrari, M Sampietro, and G Gomila, Appl Phys Lett 91 (2007) 243110.

[18] L Fumagalli, G Ferrari, M Sampietro, and G Gomila, Nano Lett 9 (2009) 1604-08.

[19] K Kathan-Galipeau, S Nanayakkara, M Nikiforov, P O’Brien, B Discher, and D Bonnell, Nano 5 (2011) 4835-42.

[20] A Plant, M Gueguetchkeri, and W Yap, Biophys J 67 (1994) 1126-33.

[21] D De Nobel and D Hofman, Physica XXII (1956) 252.

[22] RS Kane, S Takayama, E Ostuni, DE Ingber, and GM Whitesides, Biomaterials 20 (1999) 2363-76. 
